\title{
Introduction to Magnetic Resonance Imaging for Neurologists
}

Ernst-Wilhelm Radue, MD; Matthias Weigel, PhD; Roland Wiest, MD; Horst Urbach, MD

\begin{abstract}
Purpose of Review: In neuroradiology, highly sophisticated methods such as MRI are implemented to investigate different entities of the central nervous system and to acquire miscellaneous images where tissues display varying degrees of characteristic signal intensity or brightness. Compared to x-ray, CT, and ultrasound, MRI produces clearer images of tissues, body fluids, and fat. The basics of MRI may be unknown to neurologists; this article introduces MRI physics, techniques, and interpretation guidelines.

Recent Findings: This article discusses the basics of MRI to provide clinicians with the scientific underpinning of MRI technology and to help them better understand image features and improve their diagnosis and differential diagnosis by combining MRI characteristics with their knowledge of pathology and neurology.

Summary: This article will help neurologists deepen their knowledge and understanding of MRI by introducing the basics of MRI physics, technology, image acquisition, protocols, and image interpretation.
\end{abstract}

Continuum (Minneap Minn) 2016;22(5):1379-1398

\section{INTRODUCTION}

This article provides a short, but comprehensive, overview of MRI for neurologists, including the physics underlying this imaging modality, the basics of image acquisition, and the approach to imaging interpretation with MRI.

\section{A PRIMER ON MRI PHYSICS}

MRI is a noninvasive imaging modality that provides excellent soft tissue contrast for normal and pathologic structures of tissues such as cartilage, muscles, brain, and spinal cord as well as fat and body fluids. Medical imaging modalities often produce image contrasts that are related to the different absorption and reflection of waves in body tissues (eg, x-ray, CT, ultra- sound). However, MRI produces images that are related to the magnetic properties of, and molecular interactions within, the tissues under observation. Since these interactions are complex and manifold, MRI has become a powerful and versatile imaging modality that can distinguish a large number of very different tissue contrasts.

\section{Important Physical Features That Create MRI Images}

The physics of MRI can be understood best by following a number of steps and thereby investigating the underlying physics and technology. These steps also reflect the physics of an MRI measurement cycle.

Polarization. The human body consists of approximately $10^{27}$ molecules,
Address correspondence to Dr Ernst-Wilhelm Radue, Biomedical Research and

Training, University Eye Hospital Basel, Mittlere Strasse 91, CH-4031 Basel, Switzerland, ernst-wilhelm.radue@usb.ch.

Relationship Disclosure:

Dr Radue has served as a consultant for Neurologische und Psychiatrische

Universitäts Klinik Basel, has received personal compensation for speaking engagements from Novartis AG and Sanofi Genzyme, and receives royalties from

Springer-Verlag GmbH Dr Weigel has received personal compensation for speaking engagements from the European Society for

Magnetic Resonance in Medicine and Biology, the Magnetic Resonance Compact University Hospital Erlangen, and Vereinigung

Südwestdeutscher

Radiologen und

Nuklearmediziner and

royalties from the University Medical Center

of the University of Freiburg

for a patent for dynamic motion correction in MRI. Dr Wiest receives research/ grant support from the Swiss National Foundation. Dr

Urbach serves as coeditor of Clinical Neuroradiology and on the editorial board of Neuroradiology and has received personal compensation for speaking engagements from Bracco, Stryker, and UCB SA.

Unlabeled Use of

Products/Investigational Use Disclosure:

Drs Radue, Weigel, Wiest, and Urbach report no disclosures. C 2016 American Academy of Neurology. 


\section{CONTINUUM Introduction to MRI}

\section{KEY POINT}

- MRI produces images that are related to the magnetic properties of and molecular interactions within, the tissues under observation. which is an unfathomably large number. Each molecule consists of a few to thousands of atoms. Many nuclei of these molecule-bound atoms rotate around their axis; they have a so-called spin. $^{1-3}$ Spinning nuclei always generate a nuclear magnetic (dipole) moment. Thus, each spinning nucleus behaves like a tiny bar magnet with a magnetic north pole and south pole. It has become common in MRI literature to refer to these nuclear magnets in a simplified way as spins. ${ }^{1-3}$ Because of their high natural abundance in the human body and because they have the strongest magnetic moment, the nuclei of hydrogen atoms are commonly used for clinical MRI. A hydrogen nucleus consists of only one proton. Therefore, the following discussion focuses on spins of hydrogen nuclei/protons.

Although the human body contains roughly $10^{27}$ magnetic hydrogen nuclei, ie, spins, humans do not seem to be magnetic. Why? Because all the spins are randomly orientated in the body; they cancel each other, and therefore their net magnetization is $0 .{ }^{1-3}$

The basic idea of MRI is to put the patient into a very strong and static magnetic field, the so-called main field $\mathrm{B}_{0}$. This static $\mathrm{B}_{0}$ field is generated by the MRI scanner and is approximately 30,000 to 140,000 times stronger than the earth's magnetic field. As a consequence, the spins begin to orient in space and to align with the $\mathrm{B}_{0}$ field. However, compared to compass needles in the earth's magnetic field, spins align not only parallel but also antiparallel to the external static $\mathrm{B}_{0}$ field. ${ }^{1-3}$ The numbers are almost equal; only a tiny excess of spins is additionally aligned parallel to the $\mathrm{B}_{0}$ field, leading to a tiny but measurable macroscopic net magnetization, or $\mathrm{M}_{0}$. Since this effect of so-called polarization is so weak, MRI is an imaging modality that permanently suffers from low signal (and low signal to noise ratio, as described later in this article).

Excitation and precession. Initially, at rest, the net magnetization resides parallel to the $\mathrm{B}_{0}$ field in the equilibrium position; this is called the equilibrium magnetization $\mathrm{M}_{0} \cdot{ }^{1-3}$ However, an MRI scanner is able to generate a second magnetic field, $\mathrm{B}_{1}$, that oscillates in the radiofrequency range. The spins are able to absorb energy from this $\mathrm{B}_{1}$ field, and, thus, they are tipped or flipped out of the equilibrium position. This process is called excitation; the spins and, accordingly, the net magnetization are excited. ${ }^{1-3}$ The angle by which the spins/magnetization are rotated is termed the flip angle. Flip angles are usually between 0 degrees and 90 degrees.

As soon as the spins leave the equilibrium position, they start to undergo a rotating motion around the external $\mathrm{B}_{0}$ field called precession, much like a spinning top under the effect of gravity. The precession frequency of the spins is depicted by the Larmor equation. $^{1-3}$ The Larmor equation states that the precession frequency is directly proportional to the strength of the magnetic field. Thus, the higher the magnetic field, the higher the precession frequency (ie, the faster the spins rotate). Contrary to this, the lower the magnetic field, the lower the precession frequency and the slower the spins rotate.

The basic statement of the Larmor equation would not be of such great importance in MRI if all spins experienced the same magnetic field as determined by the nominal value of the scanner's $\mathrm{B}_{0}$ field (eg, $1.5 \mathrm{~T}$ or $3 \mathrm{~T}$ ) and therefore automatically had the same Larmor frequency. This is not the case for several reasons. First, the $\mathrm{B}_{0}$ main field of an MRI scanner can never be built perfectly constant in 
space as it has spatial inhomogeneities. Second, human body tissues also generate a tissue-characteristic magnetization called susceptibility, which distorts the external $\mathrm{B}_{0}$ field. Hence, the spin's Larmor frequency slightly changes with position due to $\mathrm{B}_{0}$ inhomogeneity and tissue susceptibility effects. ${ }^{1-3}$ The third reason is that an MRI scanner can generate spatially varying magnetic fields that are used for spatial encoding. These so-called gradient fields are discussed later in the article.

So far, it has been roughly said that the $\mathrm{B}_{1}$ excitation field oscillates in the radiofrequency range; however, to be more accurate, the $\mathrm{B}_{1}$ oscillation frequency has to match quite precisely the Larmor precession frequency of the spins. The two frequencies have to be in resonance. Otherwise, the spins cannot absorb the radiofrequency energy, and they are not excited. ${ }^{1-3} \mathrm{~A}$ good analogy for this observation of mandatory resonance is a child's swing. The child must move synchronously or in resonance with the swing to increase its amplitude. The modality's full name, written out as nuclear magnetic resonance imaging, directly originates from this effect. ${ }^{1-3}$

Magnetization is a vector quantity, ie, it possesses a magnitude and a direction (Figure 1-1). As has already been pointed out, the initial direction of the net magnetization is parallel to the external $\mathrm{B}_{0}$ field. By definition, this is the $\mathrm{z}$-axis of the scanner's coordinate system; it is also called the longitudinal direction. Orthogonal to the longitudinal $\mathrm{z}$-direction is the transverse plane, spanned by the orthogonal x-axis and y-axis. In MRI, any magnetization vector is decomposed into a longitudinal and a transverse component (Figure 1-1).

Measurement with radiofrequency coils. After excitation, transverse mag-

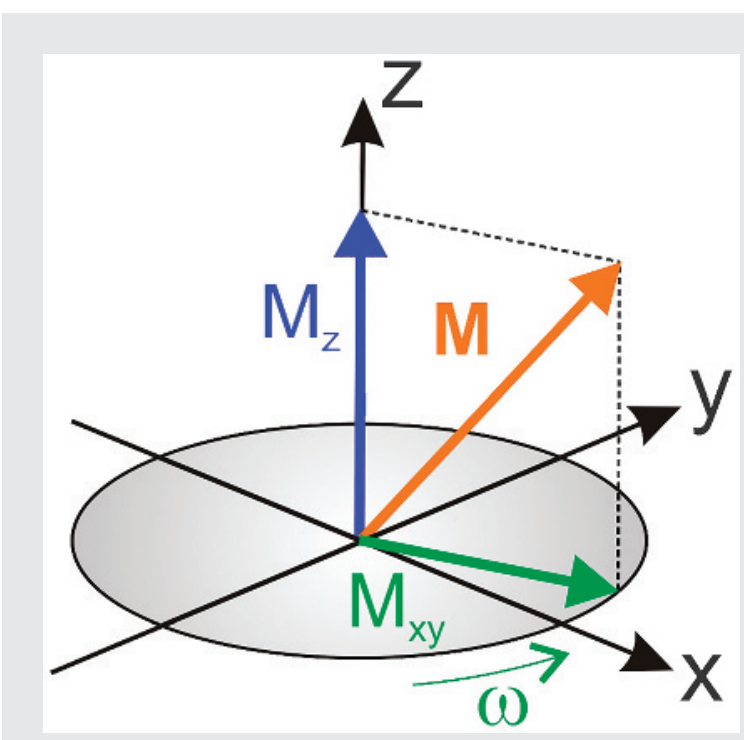

FICURE 1-1 In MRI, any magnetization vector $M$ (orange arrow) is decomposed into a longitudinal component $\mathrm{Mz}$ parallel to the z-axis (blue arrow) and into a transverse component Mxy (green arrow) in the $x-y-p l a n e$.

netization components occur and precess in the $x$-y-plane. Generally, moving or rotating magnetic moments or vectors induces electrical currents in electrical coils. Thus, the precessing excited spins induce an electrical voltage in radiofrequency receiver coils placed around the human body, similar to a generator producing electricity. ${ }^{1-3}$ Since the effect is very weak, the measured radiofrequency signal is tiny and must be amplified by a dedicated radiofrequency receiver/ amplifier chain. However, the radiofrequency coils and radiofrequency amplifier chain also measure and generate noise, which is usually of similar magnitude to the desired radiofrequency signal. For this reason, MRI inherently suffers from a low signal to noise ratio. If the MRI images become too noisy (ie, if the signal to noise ratio gets too low), diagnosis is no longer feasible.

Modern radiofrequency coils for MRI are usually close to the patient's body to improve the reception of the 


\section{CONTINUUM Introduction to MRI}

KEY POINT

- T1-weighted MRI uses $\mathrm{T} 1$ relaxation times to generate fundamental images with characteristic tissue signal intensities or tissue brightness. As a rule of thumb, the more aqueous a tissue is, the higher the $\mathrm{T} 1$ is. Fat has short T1. radiofrequency signal. Since the measured noise is directly correlated to the sensitivity volume of the radiofrequency receiver coil, small radiofrequency coils produce a higher signal to noise ratio than the scanner's large body coil. Furthermore, modern phased-array coils take advantage of this; they consist of a matrix of several small radiofrequency coils of high signal to noise ratio, combining them to be sensitive to a larger body volume. At the end of the radiofrequency receiver chain, the signal is further postprocessed by a computer to reconstruct the magnetic resonance (MR) image. ${ }^{1-3}$

Relaxation. Nature always strives for equilibrium, ie, for a uniform distribution of energy. Consequently, in MRI, the excited spins want to get rid of their energy such that the net magnetization resides as a pure longitudinal magnetization in the equilibrium position again. This effect is called relaxation. ${ }^{1-4}$ Two different types of relaxation occur in MRI, which is another reason magnetization vectors are always regarded as a longitudinal magnetization component and a transverse magnetization component.

With the equilibrium magnetization being excited, the longitudinal component is reduced (flip angle between 0 and 90 degrees) or becomes exactly 0 (a 90-degree flip angle). Then the process of longitudinal relaxation immediately starts: the longitudinal magnetization grows back to its normal size in the equilibrium state. However, this is not an immediate process, because the spins need time to transfer their excess of energy to the environment in the tissue. To be more accurate, the speed of the longitudinal relaxation is specific for a given tissue and takes place with a characteristic relaxation time $\mathrm{T} 1 .^{1-4}$ Furthermore, this $\mathrm{T} 1$ relaxation corre- sponds to an exponential asymptotic curve (Figure 1-2). Thus, T1 does not directly depict the time when the relaxation is completed, but is a measure of the speed of the relaxation or recovery of the longitudinal magnetization component (Figure 1-2).

T1 relaxation times are not only highly tissue characteristic, but they also depend on the $\mathrm{B}_{0}$ field strength of the scanner. ${ }^{1,2,4}$ They also alter with structural changes of the tissue, which makes T1 highly sensitive for pathologic changes of body tissue; T1weighted MRI uses T1 relaxation times to generate fundamental images with characteristic tissue signal intensities or tissue brightness (Figure 1-2). T1 relaxation times are roughly between half a second and a few seconds for liquids. As a rule of thumb, the more aqueous a tissue is, the higher the T1 is. Fat has short T1.

A second relaxation takes place that leads to a decay or relaxation of the generated transverse component toward 0 , which can be considerably faster than the recovery of the longitudinal component. ${ }^{1-4}$ Remember that the macroscopic net magnetization is the sum of a multitude of spins. For each spin, the Larmor equation holds true. Since the molecules and spins are in permanent thermal motion on a microscopic scale, their magnetic moments interact with each other. To put it simply, a given spin influences the local magnetic field the neighboring spins "see"; accordingly, their Larmor precession frequencies change constantly. As a consequence, the precessing transverse magnetization components of the spins get out of sync or out of phase. ${ }^{1-4}$ The individual components fan out in the transverse plane, leading to a decay of the transverse net magnetization with the characteristic and tissue-specific relaxation time T2 (Figure 1-3). The T2 


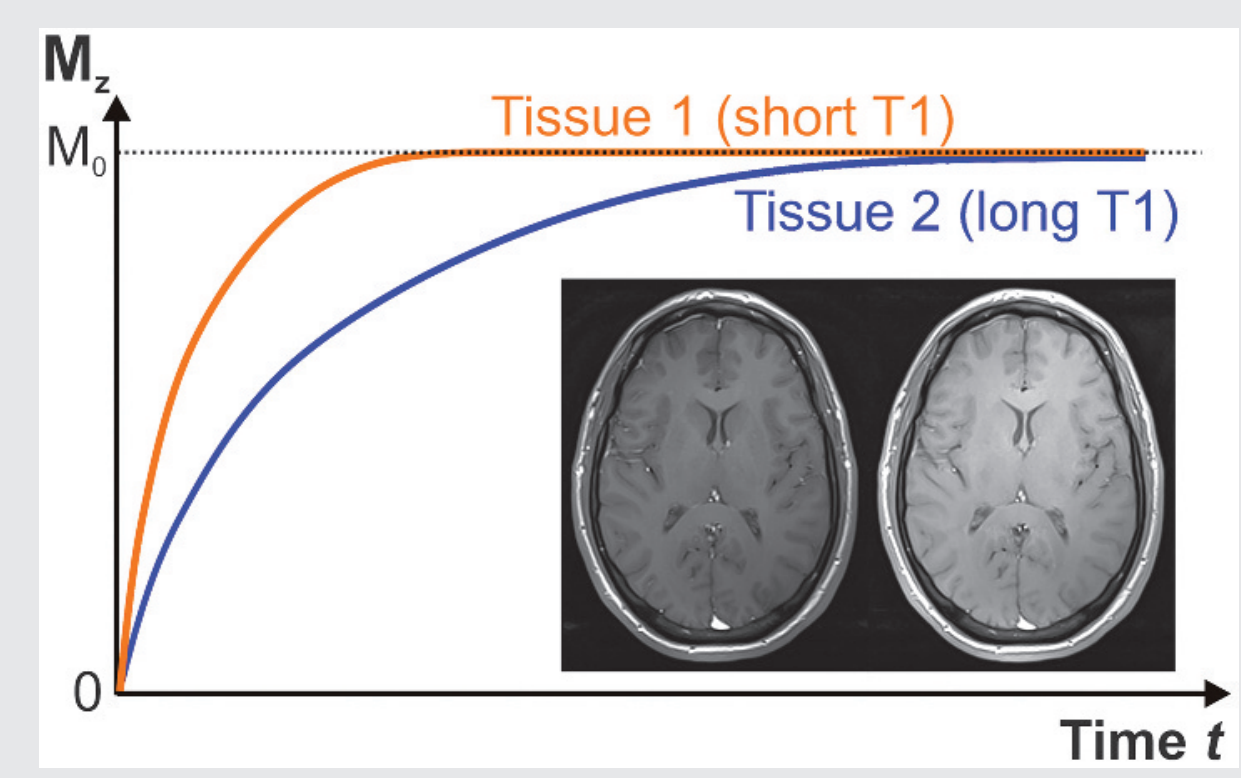

FIGURE 1-2 Longitudinal relaxation or T1 recovery curves of the (longitudinal) magnetization component $\mathrm{Mz}$ shown for two tissues with different T1 but the same proton density. In this example, tissue 1 has a short T1 (eg, body fat, orange curve), whereas tissue 2 has a long T2 (eg, CSF, blue curve). Based on such different recovery curves, T1-weighted images can be acquired; two representative examples are shown. The left image was acquired with a shorter repetition time than the right image, leading to a stronger T1 weighting. As is typical for a T1-weighted brain image, white matter is brighter than gray matter because of the shorter T1. CSF has the longest T1 and is therefore very dark.

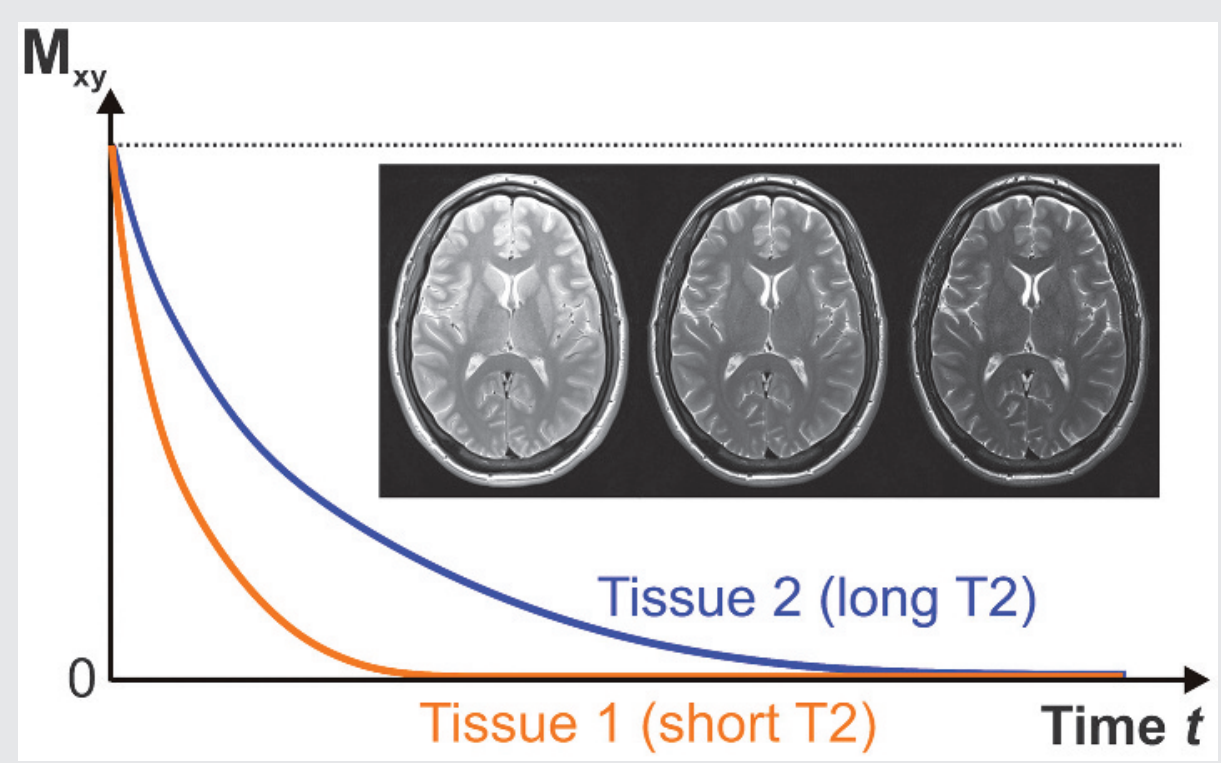

FIGURE 1-3 Transverse relaxation or T2 decay curves of the (transverse) magnetization component Mxy shown for two tissues with different $\mathrm{T} 2$ but the same proton density. In this example, tissue 1 has a short T2 (eg, body fat, orange curve), whereas tissue 2 has a long T2 (eg, CSF, blue curve). Based on such different decay curves, T2-weighted images can be acquired. Three representative examples are shown, with increasing echo time from left to right, leading to an increase in T2 weighting from left to right accordingly. As is typical for a T2-weighted brain image, gray matter is brighter than white matter because of the longer T2. CSF has the longest T2 and is therefore very bright. 


\section{CONTINUUM Introduction to MRI}

\section{KEY POINT \\ T2 alters with structural or metabolic changes of the tissue, which makes it highly sensitive for pathologic changes of body tissue; T2-weighted MRI uses T2 relaxation times to generate fundamental images with characteristic tissue signal intensities or tissue brightness.}

relaxation is an exponential decay; thus, similar to T1, T2 does not directly depict the time when the relaxation is completed but is a measure of the speed of relaxation or decay (Figure 1-3).

T2 relaxation times are approximately between 50 milliseconds and 100 milliseconds for aqueous soft tissues and roughly 1 to 2 seconds for liquids. T2 also alters with structural or metabolic changes of the tissue, which makes it highly sensitive for pathologic changes of body tissue; T2-weighted MRI uses T2 relaxation times to generate fundamental images with characteristic tissue signal intensities or tissue brightness (Figure 1-3).

Because of the random nature of the thermal motion, the spin interactions are random, and, thus, the T2 decay is irreversible. ${ }^{1-4}$ However, an additional, reversible effect leads to further decay of transverse magnetization. Inhomogeneities of the scanner's $\mathrm{B}_{0}$ field and varying susceptibility effects of the tissues, as discussed earlier in the article, also change the Larmor frequency according to the Larmor equation. These effects are fixed in space on a macroscopic scale and are, therefore, reversible by using so-called spin echoes. ${ }^{4}$ The combined relaxation of the irreversible T2 part and the reversible part is termed $\mathrm{T} 2 *{ }^{1-5}$

\section{Spatial Encoding}

So far, basic MR physics, signal generation, and measurement as well as relaxation effects have been discussed. However, to reconstruct a full image, the measured MR signal needs to be assigned to the corresponding location in the patient's body where it was generated; spatial encoding is needed. ${ }^{1-5}$ To achieve this, so-called gradient coils are employed that generate a linear magnetic field in space. In accordance with the Larmor equa- tion, the Larmor frequency also changes linearly along the gradient's direction accordingly. This effect is exploited in three ways for spatial encoding. First, during radiofrequency excitation, a gradient can be turned on, the so-called slice selection (twodimensional acquisition) or slab selection (three-dimensional acquisition) gradient. ${ }^{1-5}$ Consequently, according to the resonance condition mentioned earlier in the article, only spins are excited (and therefore generate signal) where the linearly changing precession frequencies in space match the range of radiofrequency excitation frequencies: This defines the desired slice or slab. Second, during the measurement, a gradient can be turned on, the socalled read or frequency-encoding gradient. ${ }^{1-5}$ Then, the spin locations along this read gradient are encoded into the signal via the spins' spatially dependent Larmor frequency. Third, between excitation and measurement, a gradient can be turned on, the socalled phase-encoding gradient. ${ }^{1-5}$ Thus for a duration of time, the spins along this phase-encoding gradient precess with different Larmor frequencies and therefore acquire different phases. As phases, the different angles of transverse magnetization components in the $\mathrm{x}$-y-plane are indicated (Figure 1-1). Repetitive measurement cycles with different phase-encoding gradients allow encoding spin locations into phase changes. Generally, three gradient coils also reside within the scanner, one each for the $\mathrm{x}_{-}^{-}, \mathrm{y}_{-}^{-}$, and z-direction.

The measured signal containing the spatial encoding is stored into a raw data space called $k$-space. Afterward, the main step in image reconstruction is to make use of the Fourier transform. It facilitates decoding of the k-space data such that it assigns signal intensities to their correct 
location again, resulting in the reconstructed image.

The complexity of the spatial encoding and the MR signal generation makes MRI prone to errors in these procedures, eg, caused by $\mathrm{B}_{0}$ inhomogeneities, susceptibility effects, body motion, or wrong parameter settings at the scanner. Such effects can manifest in the image as artifacts, ie, structures are displayed that do not reflect the reality, or the image may be blurred. Identifying and avoiding such artifacts is of great importance in MRI.

\section{MRI Sequences}

Generally, radiofrequency excitation, the switching of spatial encoding gradients, waiting times for relaxation, and signal measurements follow an exact and predefined timing pattern that is called the (MRI) sequence. Some parts of the sequence timing may be influenced by the user. The exact sequence timing pattern determines the characteristic brightness of the different tissues in the reconstructed images due to proton density and T1 and T2 weighting.

Dozens, hundreds, or perhaps thousands of different MRI sequences exist, depending on how the different types and variants that were published within the past 40 years are counted and classified. In practice, MRI sequences are frequently classified as being either a gradient recalled echo (GRE) or a spin echo sequence. ${ }^{4,5}$ Basically, GRE sequences use a radiofrequency excitation pulse, apply the spatial encoding, and directly measure the excited magnetization, as described earlier in the article. Spin echo sequences apply a second radiofrequency pulse after excitation and before the measurement, the so-called refocusing pulse. It reverses or refocuses the $\mathrm{B}_{0}$ inhomogeneity and susceptibility effects. Whereas spin echo sequences are more robust in signal behavior, they are slower than GRE sequences. A faster spin echo variant, the turbo or fast spin echo sequence, generates more than one echo per excitation and is one of the workhorses in clinical MRI. ${ }^{4,5}$

Important MRI sequence parameters that can usually be influenced by the user at the scanner are the echo time (TE) and the repetition time (TR). TE defines the time from the excitation until the signal is measured in the transverse plane. ${ }^{1-5}$ This is of importance because the longer the TE defined by the user, the longer the tissue-specific T2 decay takes place. Hence, the acquired image displays a stronger T2 weighting. TR defines the time between repetitive measurement cycles or excitations. ${ }^{1-5}$ If the defined TR is short compared to the characteristic T1 relaxation times of the investigated tissues, the corresponding longitudinal magnetizations cannot fully recover before the next excitation, which represents a $\mathrm{T} 1$ weighting of the magnetization. As a result, the image that is reconstructed is also T1 weighted.

$\mathrm{T} 1$ and $\mathrm{T} 2$ weighting are dominant or strong signal weightings in MRI and therefore facilitate images with excellent tissue differentiation. Morphologic acquisitions with both low T1 weighting and low T2 weighting usually bring out the third fundamental MRI weighting: proton density weighting. ${ }^{1-5}$ This weighting is proportional to the number of spins/protons in the tissue. Proton density weighting is weak compared to T1 and T2 weighting, particularly in the brain.

\section{Magnetization Preparation}

The flexibility and versatility of tissue brightness and appearance in MRI can be increased manifold by employing additional radiofrequency and gradient

\section{KEY POINTS}

Errors caused by $\mathrm{B}_{0}$ inhomogeneities, susceptibility effects, body motion, or wrong parameter settings on the scanner can manifest in the image as artifacts (structures displayed that do not reflect reality), or the image may be blurred. Identifying and avoiding such artifacts is of great importance in MRI.

Important MRI sequence parameters that can usually be influenced by the user at the scanner are the echo time and the repetition time.

Echo time defines the time from the excitation until the signal is measured in the transverse plane. This is of importance because the longer the echo time defined by the user, the longer the tissue-specific T2 decay takes place. Hence, the acquired image displays a stronger T2 weighting.

Repetition time defines the time between repetitive measurement cycles or excitations.

- If the defined repetition time is short compared to the characteristic T1 relaxation times of the investigated tissues, the corresponding longitudinal magnetizations cannot fully recover before the next excitation, which represents a $\mathrm{T} 1$ weighting of the magnetization. As a result, the image that is reconstructed is also T1 weighted. 


\section{CONTINUUM Introduction to MRI}

\section{KEY POINTS \\ - T1 and T2 weighting are dominant or strong signal weightings in MRI and therefore facilitate images with excellent tissue differentiation. \\ - Diffusion represents water motion in the tissue on a microscopic scale; it is determined by the tissue microstructure and also reflects metabolism. The resulting apparent diffusion coefficient is tissue-specific and is a measure of the mobility of the water molecules in the tissue. \\ - The principal advantage of MRI is the ability to investigate tissue-dependent responses to a magnetic field in multiple planes without the use of ionizing radiation.}

pulses. These magnetization preparations introduce dedicated weightings into the initial equilibrium magnetization before the actual excitation pulse or make the already excited magnetization sensitive to some physical effects. Both have a direct impact on the tissue brightness and appearance in the later reconstructed image.

Among important variants are inversion recovery, diffusion, flow velocity, and perfusion preparations. An inversion recovery preparation uses an initial 180-degree radiofrequency pulse to invert the longitudinal magnetization along the z-axis. Afterward, characteristic $\mathrm{T} 1$ recovery takes place toward equilibrium for all tissues; however, all longitudinal magnetizations have one moment in time when their magnitude is 0 , depending on the tissue's T1. If the excitation follows in such a moment, the corresponding tissue appears dark in the image (tissue nulling). Important types are short tau inversion recovery (STIR), in which fat is dark, and fluidattenuated inversion recovery (FLAIR), in which fluids such as CSF are dark.

By using particular gradient patterns, MRI sequences can also be made sensitive to motion effects, such as diffusion and flow in the tissue. ${ }^{6}$ These particular gradient patterns alter the phase of transverse magnetization components in dependence of motion. Diffusion represents water motion in the tissue on a microscopic scale; it is determined by the tissue microstructure and also reflects metabolism. ${ }^{6}$ The resulting apparent diffusion coefficient (ADC) is tissue-specific and is a measure of the mobility of the water molecules in the tissue. Diffusional motion is exceptionally sensitive to pathologic tissue changes, and, thus, diffusion-weighted imaging (DWI) is extremely well suited as an early marker for stroke imaging. Since diffu- sional motion is of a random nature, the introduced phase change is different for each individual spin; hence, the net transverse magnetization is attenuated. ${ }^{6}$ By contrast, flow in a vessel is a coherent motion; thus, the introduced phase change for each individual spin is the same and proportional to its speed. Consequently, flow velocities can be measured from the phase change of the net transverse magnetization.

Direct perfusion-weighted imaging is also feasible by administering contrast agents to the patient. MRI contrast agents cause a strong reduction of the local $\mathrm{T} 1$ and $\mathrm{T} 2$ relaxation times in the blood and where the contrast agent molecules are able to diffuse, which may also depend on the whether the blood-brain barrier is intact. Additional postprocessing techniques and models allow quantitative perfusion values to be derived. Prominent examples of perfusion-weighted imaging are dynamic contrast-enhanced imaging (T1-weighted) and dynamic susceptibility contrast (T2-weighted) imaging. Generally, apart from perfusionweighted imaging, contrast agents may add further information about tissue damage and are able to brighten up vessels in the body.

\section{IMAGE ACQUISITION AND MAGNETIC RESONANCE PROTOCOLS}

The principal advantage of MRI is the ability to investigate tissue-dependent responses to a magnetic field in multiple planes without the use of ionizing radiation. Basic knowledge about the brain's anatomy and typical landmarks is mandatory for reading MRI.

To acquire the appropriate slicing localizer scans using fast echo, acquisitions in three orthogonal planes are recommended. Axial imaging acquisitions should be placed in the 
midsagittal line oriented along the anterior and posterior commissure or alternatively along the genu and splenium of the corpus callosum. Coronal acquisitions should be oriented along the principle targets of the examination, ie, in an 18- to 30-degree angulation perpendicular to the long axis of the hippocampus to assess temporal lobe atrophy or mesiotemporal sclerosis, along the pituitary stalk in cases of sellar pathology, or along the main axis of the optic nerves in orbital pathology. Sagittal slices should be oriented along the midline of the corpus callosum. The appropriate sequences should follow predefined and standardized protocols. However, during acquisition, changes may be necessary to follow unexpected pathologies, and one should not rely on protocols that may be hampered by inadequate slice thickness or lack of DWI, GRE/T2*, or susceptibilityweighted imaging (SWI) sequences.

For standard acquisitions, the authors recommend DWI/ADC, T1-weighted, T2-weighted, and FLAIR sequences as a basic standard, with optional acquisitions of time-of-flight, SWI, and T1weighted gadolinium sequences with a spatial resolution between $3 \mathrm{~mm}$ and $5 \mathrm{~mm}$ (depending on field strength) with at least one high-resolution threedimensional acquisition with isotropic voxels (each side of the voxel equals the same length, here $1.0 \mathrm{~mm} \times 1.0 \mathrm{~mm} \times$ $1.0 \mathrm{~mm}$ ) (Table 1-1).

A stroke protocol for MRI should encompass DWI, FLAIR, T2*/SWI, dynamic susceptibility contrast perfusion, and time-of-flight or first-pass gadolinium MR angiography (MRA). In cases of hemorrhage, additional T1-weighted sequences may be helpful to inform about the onset of the hemorrhage. Venous time-of-flight angiograms and FLAIR and T1-weighted sequences with and without gadolin- ium detect venous thrombosis; T1and T2-weighted fat-suppressed sequences along the cervical arteries detect dissections of the arteries. Vascular malformations require timeresolved ultrafast angiograms of the affected vessels with a temporal resolution greater than 1.0 second.

Specialized high-resolution protocols are also recommended when searching for epileptogenic lesions in patients with (focal) epilepsy syndromes, since conventional protocols may miss the abnormalities in approximately $50 \%$ of cases. ${ }^{7}$ Six sequences are considered as essential, encompassing T1-weighted, high-resolution T1weighted, T2/STIR, FLAIR in coronal and axial acquisitions, and GRE/SWI sequences of $3 \mathrm{~mm}$ or more. In patients in whom there is a concern for leptomeningeal inflammation and infiltration, standard routine protocols may be complemented by postcontrast FLAIR. For differentiation between inflammatory pseudotumoral lesions and CNS tumors, DWI, perfusion imaging, and MR spectroscopy may be of additional value (Figure 1-4).

Beyond lesion analysis, standard clinical routine MRI protocols embody a wealth of information about the functional state of the brain. DWI, for example, is now part of most routine protocols. Diffusion is restricted in tissue with a dense cellular matrix or in the presence of cytotoxic edema with subsequent lowering of the ADC (Figure 1-5).

While DWI is frequently employed to detect the core of an ischemic stroke $^{8}$ or a brain abscess, ${ }^{9}$ it may also aid in the differential diagnosis between seizure-related diffusion restriction and an ischemic stroke, ${ }^{10}$ in tumefactive multiple sclerosis lesions, ${ }^{11}$ in glioma and lymphoma, ${ }^{12}$ and in the prognostic assessment of coma ${ }^{13}$ and traumatic brain injury. ${ }^{14,15}$ 


\section{CONTINUUM Introduction to MRI}

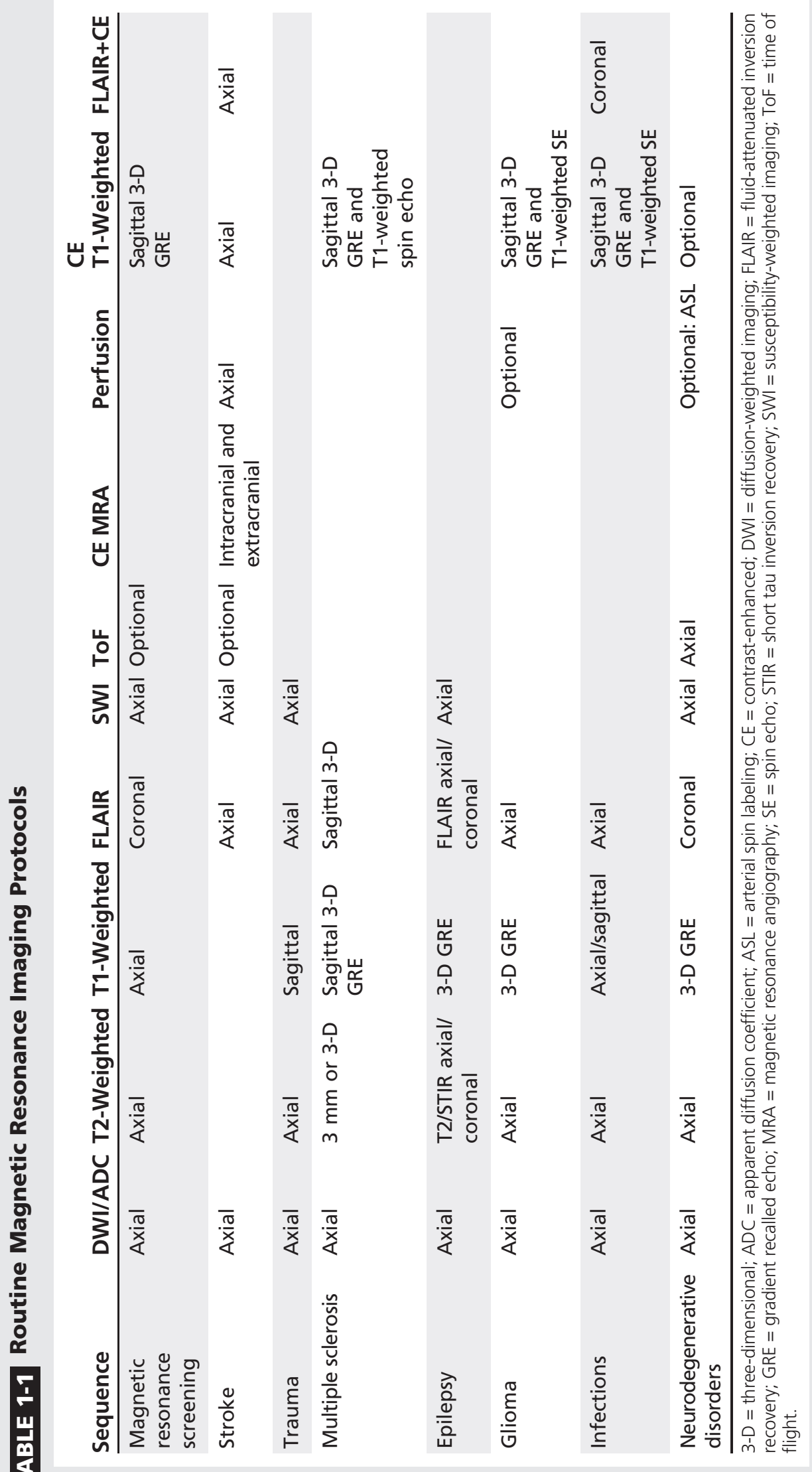




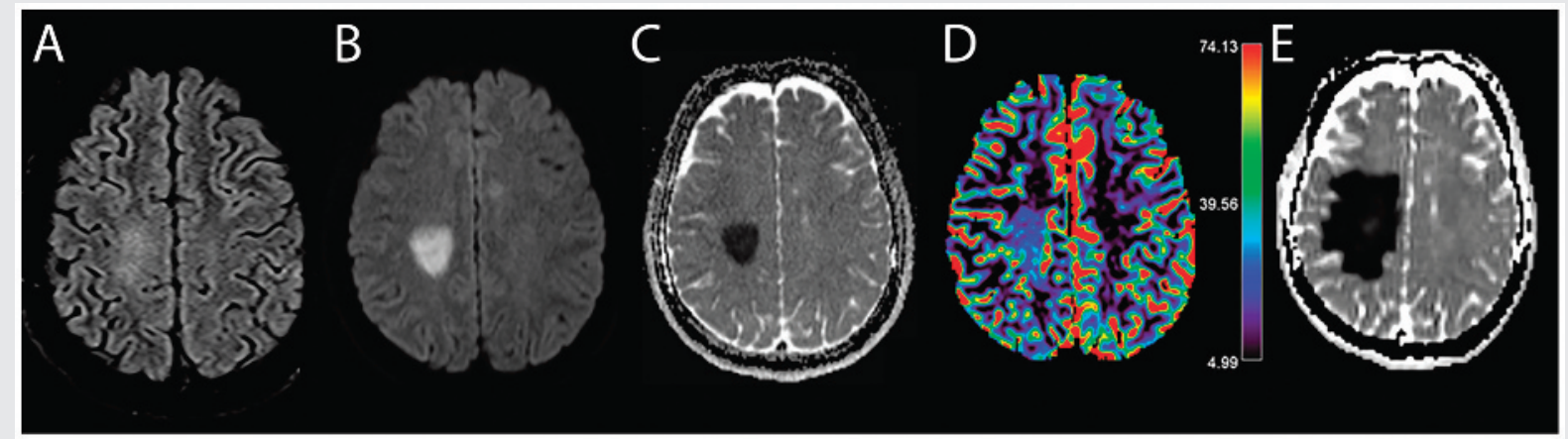

FIGURE 1-4 Signal characteristics of a tumefactive demyelinating lesion. $A$, Faint fluid-attenuated inversion recovery (FLAIR) hyperintensity; $B, C$, diffusion restriction and decreased apparent diffusion coefficient (ADC) imaging. $D$, Cerebral blood flow maps indicate hyperperfusion. $E$, Follow-up MRI (ADC map) after 24 hours indicates lesion expansion and ADC signal drop following acute demyelination.

MRI may aid also in the differentiation between vasogenic tumoral edema and tumor infiltration, ${ }^{16}$ in treatment selection of patients experiencing wake-up strokes with undefined onset, ${ }^{17}$ and in assessment of various CSF pathologies, such as meningitis, meningeal carcinomatosis, stroke, and hemorrhage ${ }^{18}$ (Figure 1-6).

SWI is used to assess disorders associated with hemoglobin breakdown (eg, parenchymal hemorrhage, cerebral amyloid angiopathy, cavernous malformations, subarachnoid hemor- rhage), calcifications, hemorrhagic contusions in traumatic brain injury, or altered blood oxygenation due to stroke or status epilepticus (Figure 1-7). ${ }^{19-20}$

Perfusion imaging in acute stroke has been shown to be a good predictor of the ischemic penumbra ${ }^{21}$ but may also aid in the differentiation between ischemic and postictal hypoperfusion ${ }^{22}$ or in migraine attacks with atypical aura symptoms. ${ }^{23}$ Typical perfusion findings in different clinical scenarios can be seen in Figure 1-8. In neuro-oncology, cell density and prominent vascularization reflect

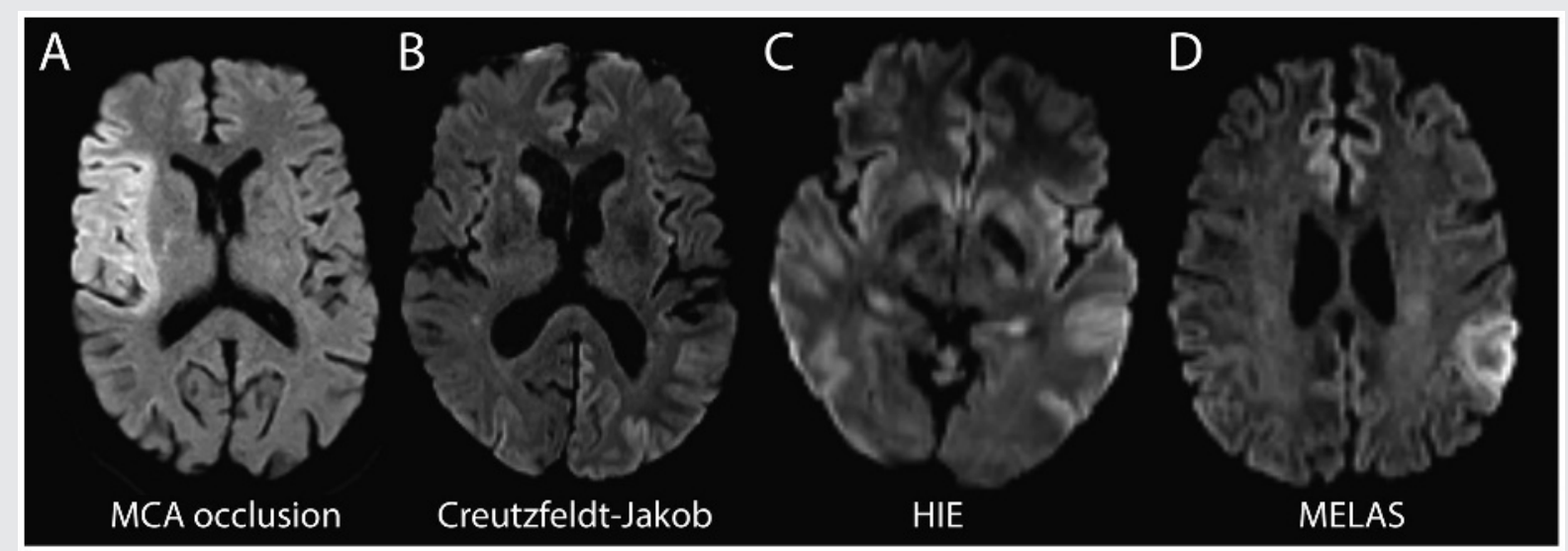

FIGURE 1-5 Patterns of diffusion-weighted imaging (DWI) restrictions in various neurologic disorders. A, Right middle cerebral artery (MCA) territory infarction; $B$, Creutzfeldt-Jakob disease (cortical, basal ganglia); $C$, symmetrically cortical in hypoxic-ischemic encephalopathy (HIE); $D$, cortical mixed cytotoxic and vasogenic pattern in mitochondrial encephalomyopathy, lactic acidosis, and strokelike episodes syndrome (MELAS). 


\section{CONTINUUM Introduction to MRI}

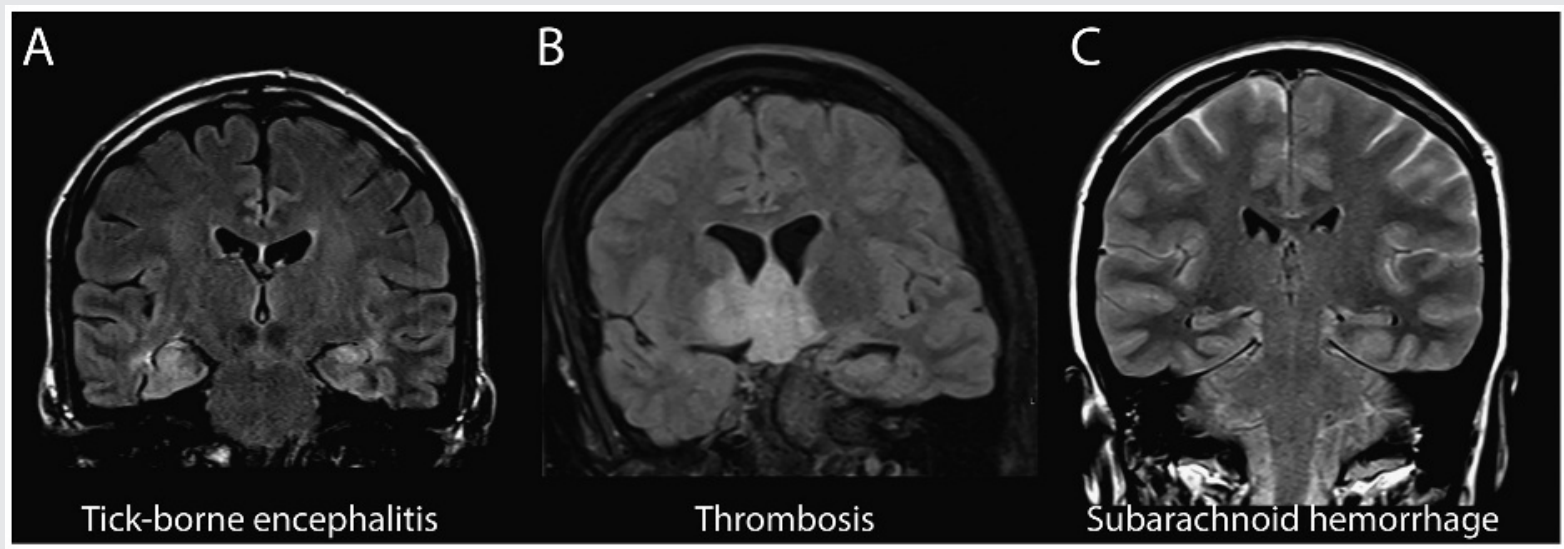

FIGURE 1-6 Coronal fluid-attenuated inversion recovery (FLAIR) imaging indicating three characteristic patterns of hyperintensity. A, Bilateral hippocampal and parahippocampal swelling in tick-borne encephalitis; $B$, bithalamic and diencephalic edema due to venous thrombosis of the internal cerebral veins; $C$, dirty CSF sign in a patient with subarachnoid hemorrhage (SAH). Note the hyperintensity due to expansion of blood into the sulcal space.

heterogeneous patterns of malignancy than can be prognostically assessed by combinations of DWI and perfusion imaging $^{24}$ to differentiate tumor progression and therapy-related effects. ${ }^{25}$

\section{GENERAL CONSIDERATIONS FOR IMAGE INTERPRETATION}

The way MRI is used in clinical practice strongly depends on the clinical needs and context of application, whether as an emergency MRI within
10 to 15 minutes because of a stroke or in an otherwise uncooperative patient, or as an elective structural or functional imaging study, which may last around 1 hour and requires extensive postprocessing procedures afterward. The underlying disorder may be misdiagnosed if, for example, an uncooperative patient is studied with a rigidly applied stroke protocol in an emergency setting. Imaging findings may be so subtle that only with the

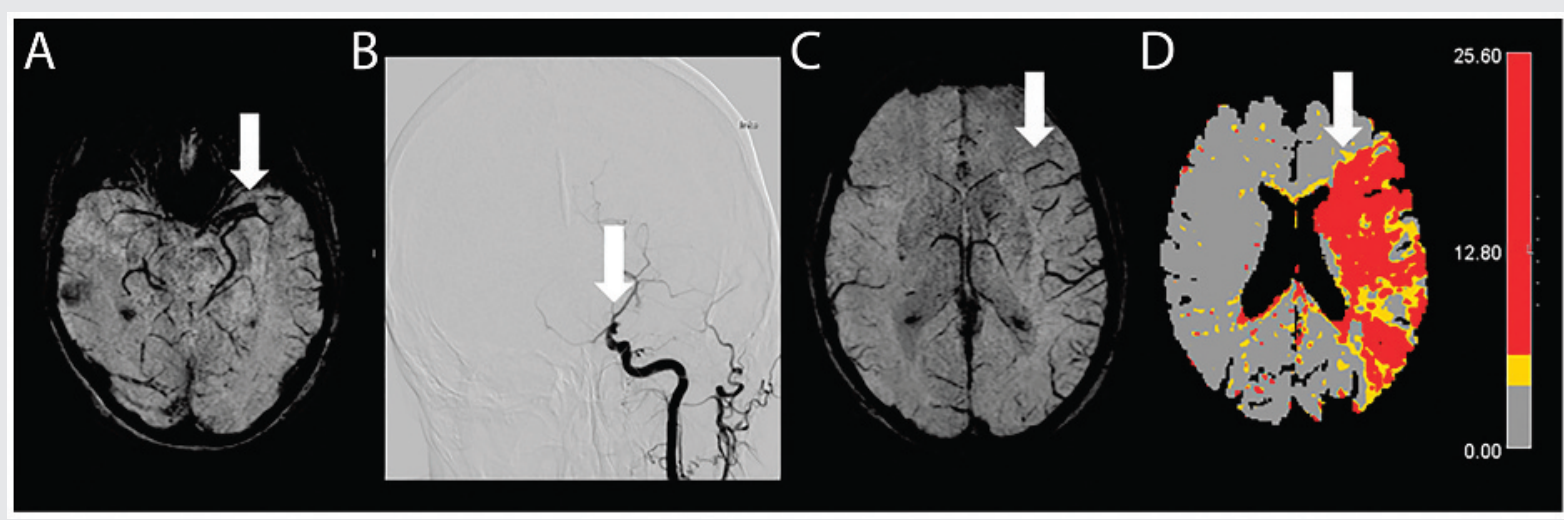

FIGURE 1-7 Advanced MRI applications for ischemic stroke. A, Axial susceptibility-weighted imaging (SWI) indicates location and thrombus length in the left middle cerebral artery (arrow); $B$, digital subtraction angiography indicates vessel occlusion of the left internal carotid artery (arrow); C, SWI indicates prominent veins due to increased oxygen extraction (paramagnetic effect) (arrow); $D$, perfusion imaging ([time-to-maximum; max delay] longer than $6 \mathrm{~s}$ indicated in red) (arrow) corresponds to the area with prominent vessels on SWI. 


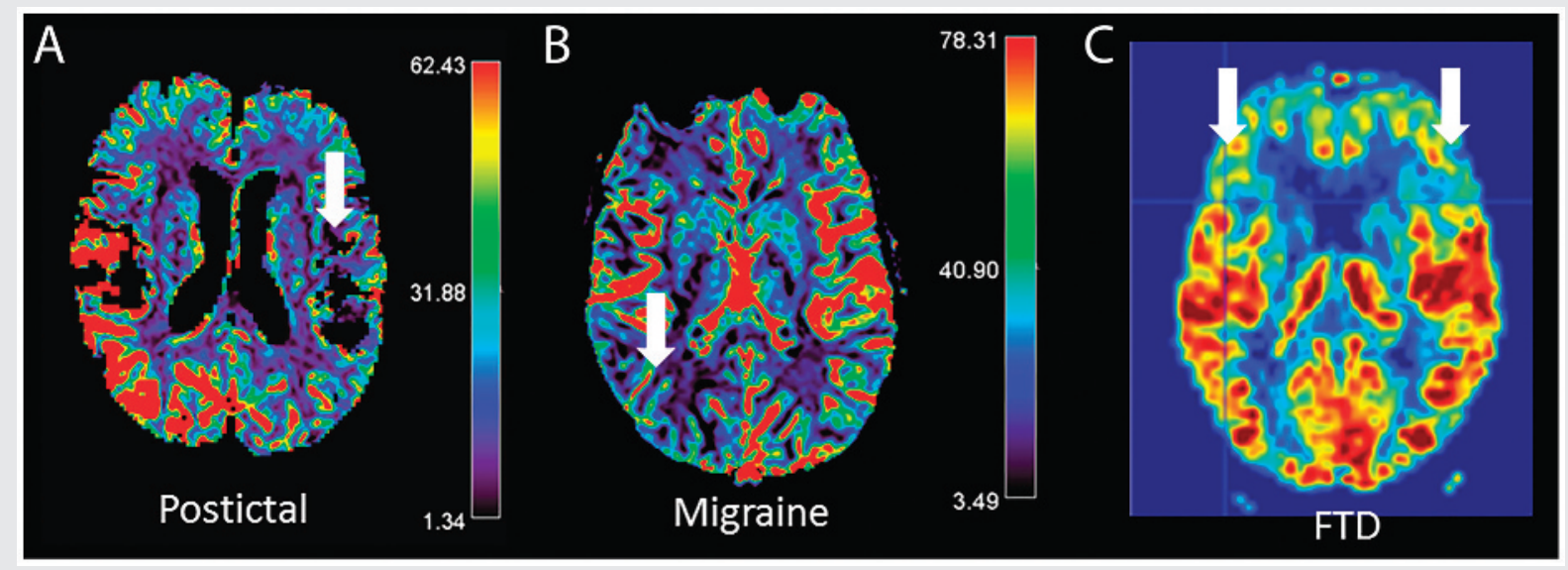

FICURE 1-8 Perfusion imaging indicating abnormal cerebral blood flow maps in different neurologic disorders. A, Patient with postictal hypoperfusion in the left temporal lobe presenting with aphasia. $B$, Patient with hypoperfusion (exceeding the vascular territories) in the right temporal and occipital lobe during a migraine attack with

headache. C, Patient with clinical presentation of frontotemporal dementia and a symmetric pattern of reduced cerebral blood flow in the frontal lobes. Arrows indicate brain areas with regional perfusion decrease.

appropriate clinical information will a lesion be found or correctly interpreted (Figure 1-9).

To interpret images correctly, reading and analysis of the MRI should incorporate a systematic approach. This may encompass a structured sequence of reading, first inspecting the DWI/ADC sequences (especially in cases where the main findings can be expected from facilitated or restricted diffusion), then switching to T2/FLAIR sequences and T1-weighted sequences with and without contrast to get a first overview of suspected pathologies. In a second step, the remaining sequences should be analyzed in the context of the suspected pathology, eg, perfusion imaging, SWI, and vascular sequences if a vascular disorder is suspected. A systematic approach should consider fixed reading
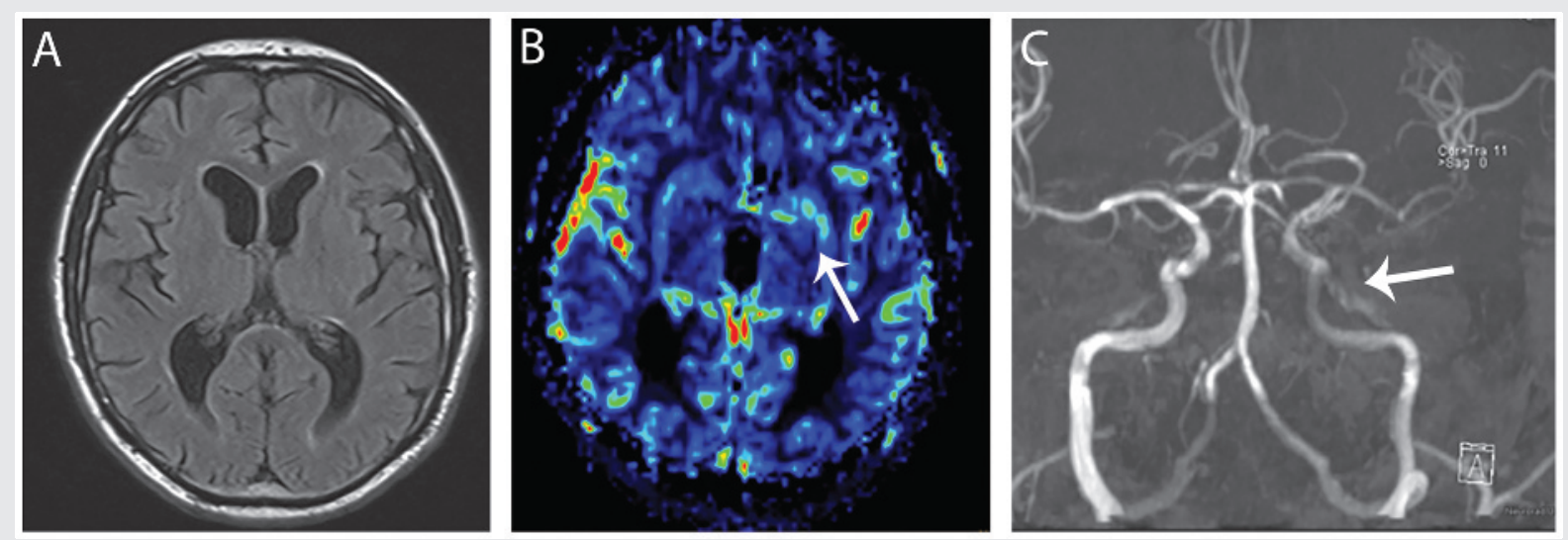

FIGURE 1-9 Imaging of a 76-year-old man with right-sided hemichoreic movements for several weeks. $A$, Axial fluid-attenuated inversion recovery (FLAIR) sequence is unremarkable. $B$, Axial cerebral blood volume map shows a slight elevation in cerebral blood volume in the left lentiform nucleus (arrow). C. Axial three-dimensional time-of-flight magnetic resonance angiogram (MRA) shows diminished signal of the left internal carotid (arrow) and middle cerebral artery suggesting a high-grade proximal internal carotid artery stenosis. 


\section{CONTINUUM Introduction to MRI}

\section{KEY POINTS}

- A systematic approach should consider fixed reading procedures, for example, from inside to outside or vice versa, from gray matter to white matter and CSF, or from suspected pathology to general inspection on a slice-by-slice basis.

- If a lesion is detected, it should first be classified by broad category, eg, tumoral lesion, vascular pathology, infection, or degeneration. procedures, for example, from inside to outside or vice versa, from gray matter to white matter and CSF, or from suspected pathology to general inspection on a slice-by-slice basis. It cannot be emphasized enough that the peripheral parts of the images need to beinspected carefully; most pathologies present in the periphery may be missed if those areas are not examined carefully by the image reader. If possible, images should be read twice, either by the neuroradiologist and the neurologist or by two specialized readers, to enable consistency checks of the suspected findings, with the final goal to identify and interpret structural lesions. If a lesion is detected, it should first be classified by broad category, eg, tumoral lesion, vascular pathology, infection, or degeneration. Depending on the underlying pathology, typical presentations and imaging features may be taken into account:

- Cerebral tumors: Slowly progressive focal clinical deficit, cognitive decline, or epileptic seizures. MRI indicates intraaxial or extraaxial pathology, a space-occupying effect, gadolinium enhancement. vasogenic edema, and midline shift,

- Vascular: Sudden onset of a neurologic deficit. MRI information may include vascular territory and DWI lesion with ADC reduction.

- Infections: Headache, fever, focal neurologic deficit, or increased cells in CSF. MRI findings include swelling of cerebral sulci or meningeal enhancement.

- Degenerative diseases: Cognitive decline, epileptic seizures. MRI may indicate focal or generalized atrophy, no gadolinium enhancement, and no focal lesions.

If no lesion is found, one should carefully reanalyze the images and take into consideration that a lesion may have been overlooked. A lesion may be overlooked because of an insufficient spatial resolution or insufficient contrast to the surrounding tissue (Figure 1-10)
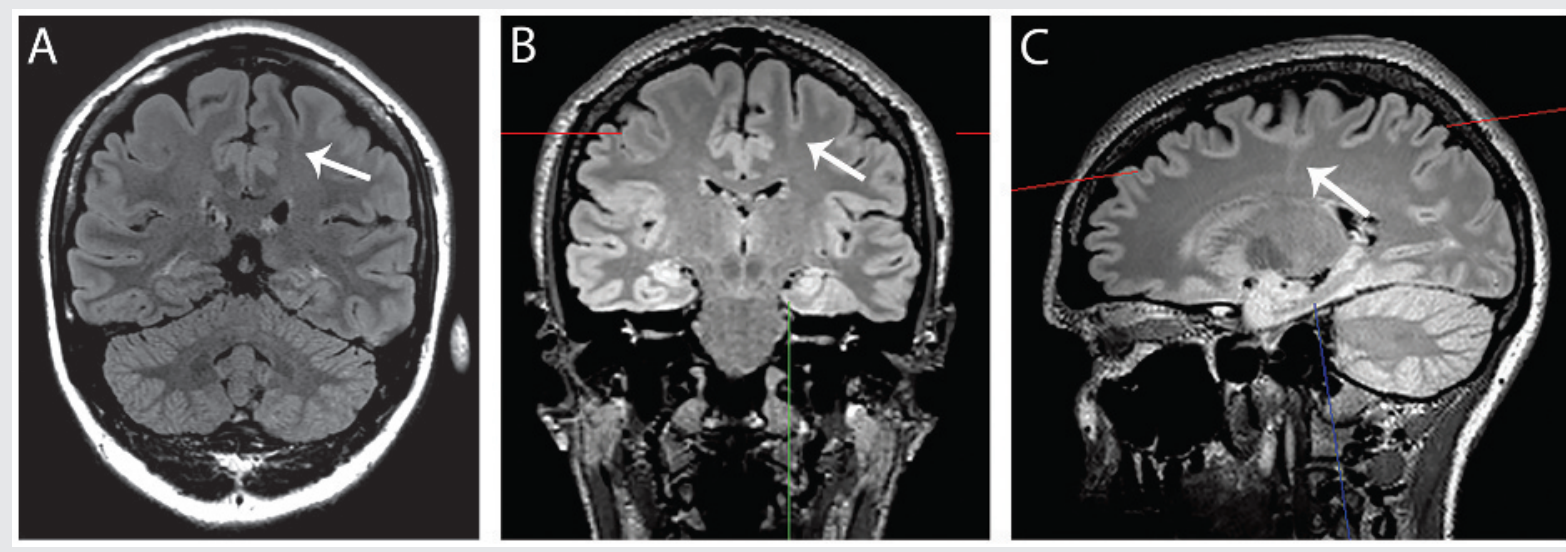

FIGURE 1-10 Focal cortical dysplasia type IIB. A, Coronal two-dimensional fluid-attenuated inversion recovery (FLAIR) turbo spin echo sequence with a slice thickness of $2 \mathrm{~mm}$; $B$, coronal reformation of a three-dimensional FLAIR sampling perfection with application-optimized contrasts using different flip angle evolutions (SPACE) sequence; $C$, sagittal three-dimensional FLAIR SPACE sequence $(1 \mathrm{~mm} \times 1 \mathrm{~mm} \times 1 \mathrm{~mm}$ voxel). Despite a higher signal to noise ratio, the funnel-shaped hyperintensity is hardly visible on the two-dimensional sequence $(A$, arrow). Multiplanar coronal reformation $(B)$ of the three-dimensional FLAIR sequence helps locate the lesion, which is typically found in the depth of a sulcus (B, $C$, arrows). 
or because of misleading clinical information or a lack of clinical information. In some instances, a lesion may not yet be visible, eg, during an early manifestation of an acute spinal infarction (Figure 1-11) or in the case of a lesion that is responsible for the clinical symptoms but hidden among many others.

Although lesion detectability is dependent on the contrast to noise ratio, ie, the signal to noise ratio of the lesion versus the signal to noise ratio of the surrounding tissue, in most cases, for a lesion to be detected, it must be at least 2 voxels (volume element defined by the slice thickness and the in-plane resolution) in size. If a lesion is less than 2 voxels in size, it may easily be missed because of partial volume effects.

Two-dimensional sequences have a higher in-plane resolution and a higher signal to noise ratio than threedimensional sequences ${ }^{26}$; however, they rarely cover the entire brain with a slice thickness of $1 \mathrm{~mm}$ to $2 \mathrm{~mm}$. Three-dimensional sequences are generally acquired in sagittal orientation

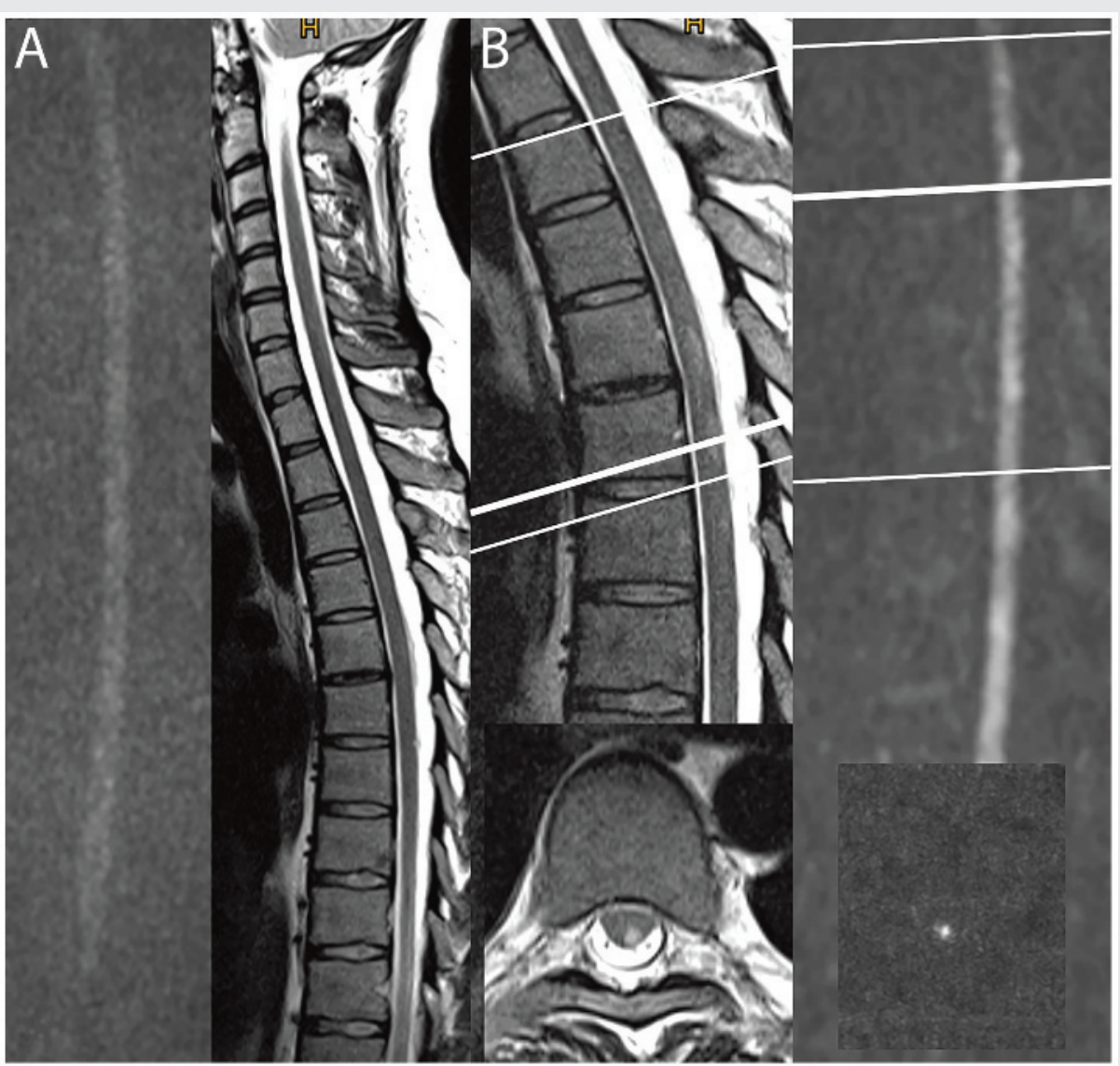

FIGURE 1-11 A 31-year-old man with sudden onset of pain and paraplegia below the level of T10. A, Initial MRI with T2-weighted fast spin echo and sagittal diffusion-weighted imaging (not shown) was unremarkable. $B$, Follow-up axial imaging 3 days later shows a left-sided posterolateral spinal infarct. The insets in panel $B$ are the axial images at the positions shown by the thick white lines in the sagittal T2-weighted fast spin echo (left) and diffusion-weighted (right) images. The thin white lines represent the range that is covered by the axial sequences. 


\section{CONTINUUM Introduction to MRI}

\section{KEY POINTS}

- Image interpretation should always depend on the combination and integration of imaging features and clinical information.

- Since location is a highly relevant parameter for diagnosis, a strong effort should be made to categorize a lesion with respect to its topography.

- The signal intensity of a lesion may provide information about its composition. A lesion is classified as hypointense, isointense, or hyperintense as compared to the gray matter if not otherwise stated.

- T1-hyperintense lesions consist of fat, blood in the methemoglobin stage, or proteinaceous fluid, or show contrast enhancement. and can be reformatted in any plane using a multiplanar reformation tool. This multiplanar reformation helps to find subtle (epileptogenic) lesions (Figure 1-10). On the other hand, high in-plane resolution may be needed to show the details of a lesion and make a specific diagnosis (Figure 1-12).

Image interpretation should always depend on the combination and integration of imaging features and clinical information. While the signal intensity may be a specific characteristic for a suspected lesion (eg, the high DWI signal of epidermoid cysts, abscesses, and infarctions), a combination of several imaging parameters are of crucial importance to narrow the differential diagnosis. A checklist of characteristic imaging features to be analyzed ahead of image interpretation are summarized in Table 1-2.

Since location is a highly relevant parameter for diagnosis, a strong effort should be made to categorize a lesion with respect to its topography. Try to exactly locate a lesion with respect to: the spaces covering the brain (epidural, subdural, subarachnoid space), cortex, white matter, ventricular ependymal, and ventricular space. The location of a lesion is appropriately assessed with the lesion cut perpendicular to its surface and considering the effect on the surroundings (Figure 1-13). In most cases, an appropriate reformation of a threedimensional sequence (eg, FLAIR, magnetization-prepared rapid acquisition gradient-echo imaging [MPRAGE]) is considered helpful.

The signal intensity of a lesion may provide information about its composition. A lesion is classified as hypointense, isointense, or hyperintense as compared to the gray matter if not otherwise stated. T1-hyperintense lesions consist of fat, blood in the methemoglobin stage, or proteinaceous fluid, or show contrast enhancement. Blood vessels typically are void of any signal (flow void); they may, however, show a paradoxical enhancement on noncontrast T1-weighted GRE sequences.

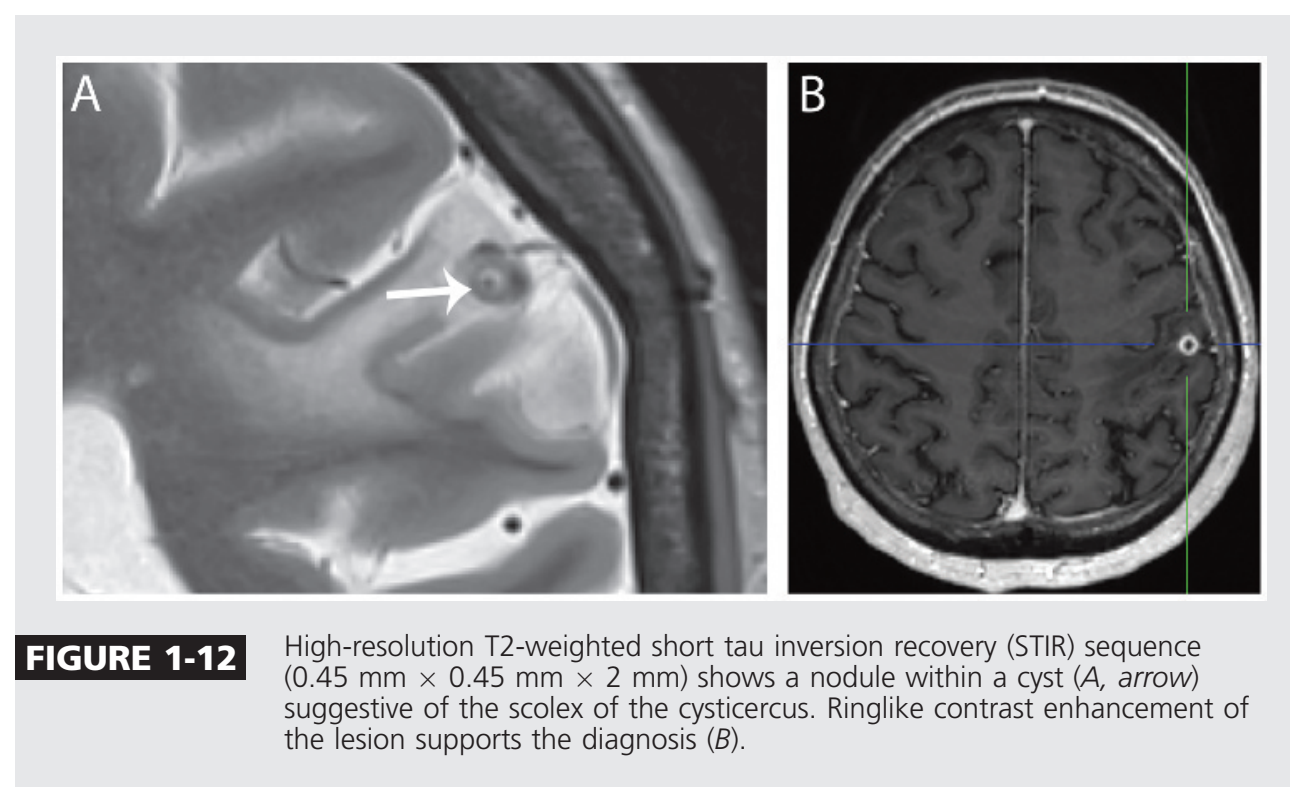




\section{TABLE 1-2 Characteristic Imaging Features to Be Analyzed Ahead of Image Interpretation}

\section{- Location}

Intraaxial, extraaxial (epidural, subdural, CSF space)

Lobe, gyrus, ventricular system

Gray matter, white matter (central, peripheral, $\mathrm{U}$ fiber involvement)

\section{- Morphology}

Exophytic component

Cystic component

Encapsulated process

Central necrosis

Calcifications

Hemorrhage

Perifocal edema

\section{- Signal Intensity}

Spin density, $\mathrm{T} 1$ relaxation, $\mathrm{T} 2$ relaxation

Susceptibility

Diffusion (water mobility)

Flow (flow void)

- Delineation

- Contrast Enhancement

Blood-brain barrier disturbance

Vessels

- Effect on Environment

Space occupying

Not space occupying

Space giving

Bone destruction

Bone remodeling

- Growth Pattern

Infiltrative

Multiplicity

Metastases
The signal intensity of lesions that are T1 isointense, T1 hypointense, and T2 hypointense, isointense, or hyperintense is related to the cellularity of these lesions. Lesions with a high cellularity (high nucleus to cytoplasm ratio) show a relatively high signal on T1-weighted images and a relatively low signal on T2-weighted images (Figure 1-14).

DWI provides information about the mobility of free water protons within a lesion, complementing SWI and T2-weighted sequences, which reveal whether a lesion contains calcification or blood degradation products. Sometimes additional application of CT may be necessary to confirm the diagnosis of a calcification or a cavernous malformation or to show the osseous structures more clearly (Figure 1-15).

Interpretation of pathologic imaging features require a fundamental knowledge of normal anatomy and signal and their variants. Careful consideration of the localization of lesions, space-occupying effects, signal abnormalities of $\mathrm{T} 1$ and $\mathrm{T} 2$, and contrast enhancements help determine whether a correct diagnosis is made based on MRI.

\section{CONCLUSION}

MRI produces images that represent signal intensities of tissue that are dependent on relaxation time and spatial resolution. Pathologic structures are identified by specific tissue behavior mainly related to different relaxation times. Combined with knowledge of clinical symptoms and signs, and recognition of suspected localization and etiology, careful assessment and interpretation of MRI scans is an essential diagnostic skill.

\section{KEY POINT}

MRI produces images that represent signal intensities of tissue that are dependent on relaxation time and spatial resolution. 


\section{CONTINUUM Introduction to MRI}

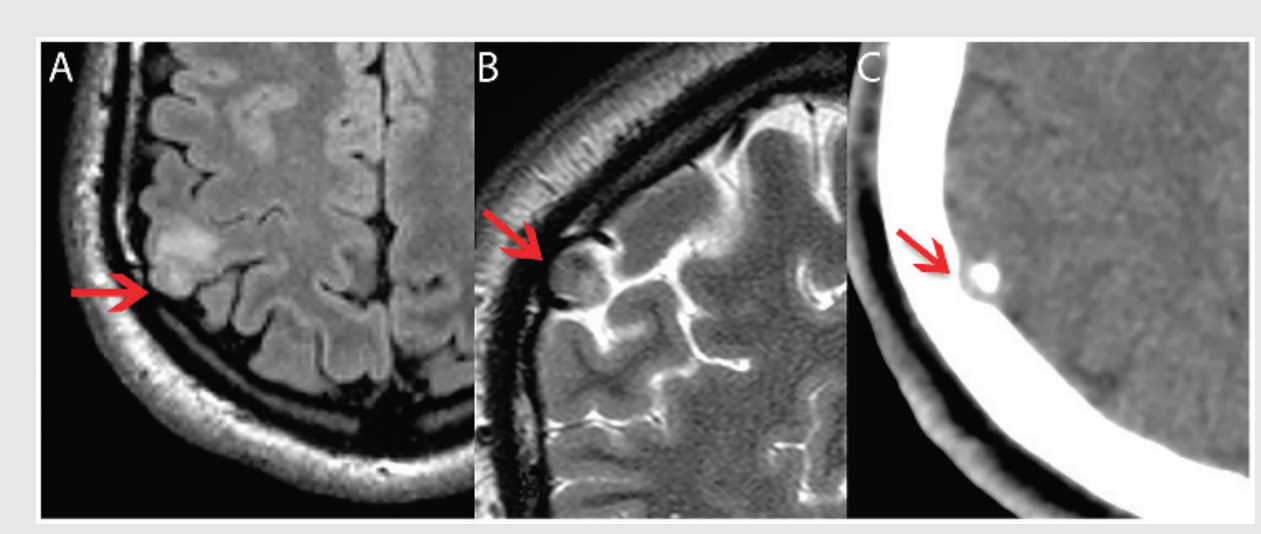

FICURE 1-13 Ganglioglioma with remodeling of the adjacent bone. A, Axial fluid-attenuated inversion recovery (FLAIR) MRI; $B$, coronal T2-weighted fast spin echo sequence; $C, C T$ shows a tiny cortical partially calcified lesion with exophytic growth/bony remodeling $(A, B, C$, arrows).
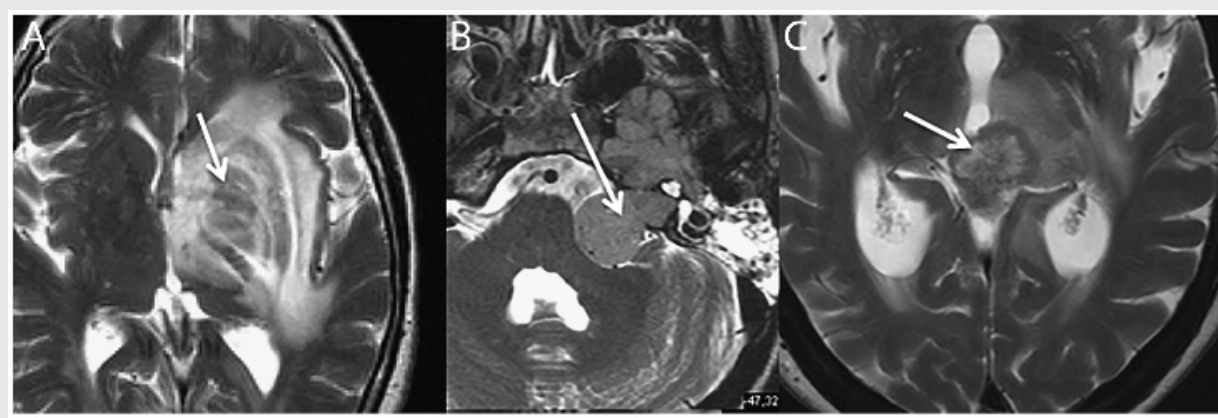

FICURE 1-14 T2 lesions with relative hypointensity suggestive for highly cellular lesions such as lymphoma ( $A$, arrow), vestibular schwannoma, or, as in this case, meningioma ( $B$, arrow) and metastasis ( $C$, arrow).

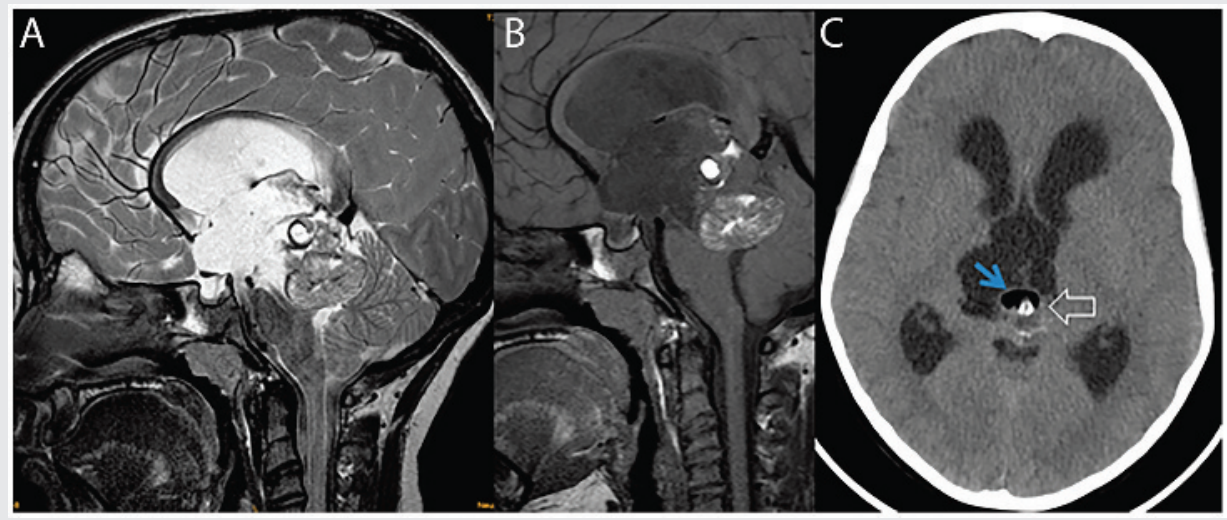

FICURE 1-15 A 12-year-old girl with a teratoma in the pineal region. A clue to the diagnosis is the mixture of fat and calcified elements on T2-weighted $(A)$ and T1-weighted $(B)$ images. Fat is hyperintense on T1-weighted and T2-weighted images; calcification may show different signal intensities. A complementary CT scan (C) helps to differentiate fat as hypodense (blue arrow) and calcification as strongly hyperdense (open arrow). 


\section{REFERENCES}

1. Horowitz AL. MRI physics for physicians. New York, NY: Springer Verlag, 1989.

2. McRobbie DW, Moore EA, Graves MJ, Prince MR. MRI from picture to proton. Cambridge, UK: Cambridge University Press, 2007.

3. Schild HH. MRI made easy. Berlin, Germany: Nationales Druckhaus \& Schering, 1990.

4. Jung BA, Weigel M. Spin echo magnetic resonance imaging. J Magn Reson Imaging 2013;37(4):805-817. doi:10.1002/ jmri.24068.

5. Markl M, Leupold J. Gradient echo imaging. J Magn Reson Imaging 2012;35(6): 1274-1289. doi:10.1002/jmri.23638.

6. Johansen-Berg $H$, Behrens TEJ. Diffusion MRI: from quantitative measurement to in vivo neuroanatomy. Cambridge, MA: Academic Press, 2013.

7. Wellmer J, Quesada CM, Rothe L, et al. Proposal for a magnetic resonance imaging protocol for the detection of epileptogenic lesions at early outpatient stages. Epilepsia 2013;54(11):1977-1987. doi:10.1111/epi.12375.

8. Albers GW, Thijs VN, Wechsler L, et al. Magnetic resonance imaging profiles predict clinical response to early reperfusion: the diffusion and perfusion imaging evaluation for understanding stroke evolution (DEFUSE) study. Ann Neurol 2006;60(5):508-517. doi:10.1002/ ana.20976.

9. Desprechins B, Stadnik T, Koerts G, et al. Use of diffusion-weighted MR imaging in differential diagnosis between intracerebral necrotic tumors and cerebral abscesses. AJNR Am J Neuroradiol 1999; 20(7):1252-1257.

10. Szabo K, Poepel A, Pohlmann-Eden B, et al. Diffusion-weighted and perfusion MRI demonstrates parenchymal changes in complex partial status epilepticus. Brain 2005;128(pt 6):1369-1376. doi:10.1093/ brain/awh454.

11. Malhotra HS, Jain KK, Agarwal A, et al. Characterization of tumefactive demyelinating lesions using MR imaging and in-vivo proton MR spectroscopy. Mult Scler 2009;15(2):193-203. doi:10.1177/1352458508097922.

12. Yamasaki F, Kurisu K, Satoh K, et al. Apparent diffusion coefficient of human brain tumors at MR imaging. Radiology 2005;235(3):985-991. doi:10.1148/ radiol.2353031338.

13. Choi SP, Park KN, Park HK, et al. Diffusion-weighted magnetic resonance imaging for predicting the clinical outcome of comatose survivors after cardiac arrest: a cohort study. Crit Care 2010;14(1):R17. doi:10.1186/cc8874.

14. Huisman TA, Sorensen AG, Hergan $K$, et al. Diffusion-weighted imaging for the evaluation of diffuse axonal injury in closed head injury. J Comput Assist Tomogr 2003;27(1):5-11.

15. Karaarslan E, Arslan A. Diffusion weighted MR imaging in non-infarct lesions of the brain. Eur J Radiol 2008;65(3):402-416. doi:10.1016/ j.ejrad.2007.04.023.

16. Artzi M, Bokstein F, Blumenthal DT, et al. Differentiation between vasogenic-edema versus tumor-infiltrative area in patients with glioblastoma during bevacizumab therapy: a longitudinal MRI study. Eur J Radiol 2014;83(7):1250-1256. doi:10.1016/ j.ejrad.2014.03.026.

17. Thomalla $G$, Cheng $B$, Ebinger $M$, et al. DWI-FLAIR mismatch for the identification of patients with acute ischaemic stroke within $4.5 \mathrm{~h}$ of symptom onset (PRE-FLAIR): a multicentre observational study. Lancet Neurol 2011;10(11):978-986. doi:10.1016/ S1474-4422(11)70192-2.

18. Stuckey SL, Goh TD, Heffernan T, Rowan D. Hyperintensity in the subarachnoid space on FLAIR MRI. AJR Am J Roentgenol 2007;189(4):913-921. doi:10.2214/ AJR.07.2424.

19. Aellen J, Abela E, Buerki SE, et al. Focal hemodynamic patterns of status epilepticus detected by susceptibility weighted imaging (SWI). Eur Radiol 2014;24(11):2980-2988. doi:10.1007/ s00330-014-3284-9.

20. Ong BC, Stuckey SL. Susceptibility weighted imaging: a pictorial review. $\mathrm{J}$ Med Imaging Radiat Oncol 2010;54(5):435-449. doi:10.1111/ j.1754-9485.2010.02208.x.

21. Olivot JM, Mlynash M, Thijs VN, et al. Geography, structure, and evolution of diffusion and perfusion lesions in Diffusion and perfusion imaging Evaluation For Understanding Stroke Evolution (DEFUSE). Stroke 2009; 40(10):3245-3251. doi:10.1161/ STROKEAHA.109.558635.

22. Hauf M, Slotboom J, Nirkko A, et al. Cortical regional hyperperfusion in nonconvulsive status epilepticus measured by dynamic brain perfusion CT. AJNR Am J Neuroradiol 2009;30(4):693-698. doi:10.3174/ajnr.A1456.

23. Förster $A$, Wenz $H$, Kerl HU, et al. Perfusion patterns in migraine with aura. Cephalalgia 2014;34(11):870-876. doi:10.1177/ 0333102414523339 . 


\section{CONTINUUM Introduction to MRI}

24. Deike K, Wiestler B, Graf M, et al. Prognostic value of combined visualization of MR diffusion and perfusion maps in glioblastoma. J Neurooncol 2016;126(3):463-472. doi:10.1007/ s11060-015-1982-z.

25. Farid N, Almeida-Freitas DB, White NS, et al. Combining diffusion and perfusion differentiates tumor from bevacizumab-related imaging abnormality (bria). J Neurooncol 2014;120(3):539-546. doi:10.1007/s11060-014-1583-2.

26. Tschampa HJ, Urbach $\mathrm{H}$, Malter $\mathrm{M}$, et al. Magnetic resonance imaging of focal cortical dysplasia: comparison of 3D and 2D fluid attenuated inversion recovery sequences at 3T. Epilepsy Res 2015;116:8-14. doi:10.1016/j.eplepsyres.2015.07.004. 